\title{
ER $\beta$ Accelerates Diabetic Wound Healing by Ameliorating Hyperglycemia-Induced Persistent Oxidative Stress
}

\author{
Xueqing Zhou ${ }^{1,2 \dagger}$, Min $\mathrm{Li}^{2 \dagger}$, Meifang Xiao ${ }^{3+}$, Qiongfang Ruan ${ }^{2}$, Zhigang $\mathrm{Chu}^{2}$, Ziqing $\mathrm{Ye}^{2}$, \\ Liyan Zhong ${ }^{3}$, Haimou Zhang ${ }^{4}$, Xiaodong Huang ${ }^{2}$, Weiguo Xie ${ }^{2 *}$, Ling $\mathrm{Li}^{3 *}$ and Paul Yao ${ }^{2,3 *}$ \\ ${ }^{1}$ Department of General Surgery, Zhongnan Hospital of Wuhan University, Wuhan, China, ${ }^{2}$ Institute of Burns, Tongren \\ Hospital of Wuhan University (Wuhan Third Hospital), Wuhan, China, ${ }^{3}$ Hainan Maternal and Child Health Hospital, Haikou, \\ China, ${ }^{4}$ State Key Lab of Biocatalysis and Enzyme Engineering, School of Life Sciences, Hubei University, Wuhan, China
}

OPEN ACCESS

Edited by:

Undurti Narasimha Das, UND Life Sciences LLC, United States

Reviewed by:

Prasad Umesh Kasbekar, Bhatia Hospital, India

Zhujiayuan Jiayuan Zhu, First Affiliated Hospital of Sun Yat-sen

University, China

*Correspondence:

Weiguo Xie

wgxie@hotmail.com

Ling $\mathrm{Li}$

Icl115@163.com

Paul Yao

vasilis112@yahoo.com

${ }^{\dagger}$ These authors have contributed equally to this work

Specialty section:

This article was submitted to

Diabetes,

a section of the journal

Frontiers in Endocrinology

Received: 19 December 2018

Accepted: 09 July 2019

Published: 24 July 2019

Citation:

Zhou X, Li M, Xiao M, Ruan Q, Chu Z, Ye Z, Zhong L, Zhang $H$, Huang $X$, Xie W, Li L and Yao P (2019) ER $\beta$ Accelerates Diabetic Wound Healing by Ameliorating

Hyperglycemia-Induced Persistent

Oxidative Stress.

Front. Endocrinol. 10:499. doi: 10.3389/fendo.2019.00499
Delayed wound healing in diabetic patients is a serious diabetic complication, resulting in major health problems as well as high mortality and disability. The detailed mechanism still needs to be fully understood. In this study, we aim to investigate potential mechanisms and explore an efficient strategy for clinical treatment of diabetic wound healing. Human umbilical endothelial cells were exposed to hyperglycemia for 4 days, then switched to normoglycemia for an additional 4 days. The cells were harvested for the analysis of reactive oxygen species (ROS) generation, gene expression and VEGF signaling pathway. Furthermore, the diabetic wound model was established in rats for the evaluation of wound healing rates under the treatment of either ER $\beta$ agonist/antagonist or SOD mimetic MnTBAP. Our results show that transient hyperglycemia exposure results in persistent ROS overgeneration after the switch to normoglycemia, along with suppressed expression of ER $\beta$, SOD2, and the VEGF signaling pathway. Either ER $\beta$ expression or activation diminishes ROS generation. In vivo experiments with diabetic rats show that ER $\beta$ activation or SOD mimetic MnTBAP diminishes ROS generation in tissues and accelerates diabetic wound healing. Transient hyperglycemia exposure induces ROS generation and suppresses ER $\beta$ expression, subsequently resulting in SOD2 suppression with additional elevated ROS generation. This forms a positive-feed forward loop for ROS generation with persistent oxidative stress. ER $\beta$ expression or activation breaks this loop and ameliorates this effect, thereby accelerating diabetic wound healing. We conclude that ER $\beta$ accelerates diabetic wound healing by ameliorating hyperglycemia-induced persistent oxidative stress. This provides a new strategy for clinical treatment of diabetic wound healing based on ER $\beta$ activation.

Keywords: ER $\beta$, SOD2, mitochondria, oxidative stress, wound healing

\section{INTRODUCTION}

Wound healing is involved with many tissues and factors, including endothelial cells, fibroblasts, and blood cells, together with many sequential process of inflammation, granulation formation and tissue remodeling. Diabetes delays wound healing due to its contribution to the defective regulation of complicated molecular and cellular events in the proper healing process (1). 
Diabetic foot ulcer (DFU) is one of the most serious diabetic complications that results from poor wound healing, which subsequently results in a major health problem in patients, causing high mortality and disability $(2,3)$. Diabetic wound healing is involved with multiple complex pathophysiological mechanisms with many extrinsic and intrinsic factors (4). Full understand of the detailed mechanism is still quite necessary for the development of an efficient clinical treatment strategy for diabetic wound healing $(5,6)$.

Hyperglycemia-induced over-generation of reactive oxygen species (ROS) and subsequent oxidative stress is a major contributor to diabetic complications, as this ROS initiates many pathological signaling pathways, resulting in diabetic tissue damage (7-9). The antioxidant enzyme SOD2 (mitochondrial superoxide dismutase) diminishes mitochondrial $\mathrm{O}_{2}$ - and plays a protective role in this process. SOD2 suppression results in increased ROS generation with subsequent mitochondrial dysfunction and oxidative stress $(10,11)$; this may be one of the reasons for delayed wound healing in diabetic patients $(12,13)$.

Estrogen receptor $\beta(\mathrm{ER} \beta)$ modulates the basal expression of SOD2, regulates oxidative stress (12) and mitochondrial function $(14,15)$, and plays a protective role in tissue damage. ER $\beta$ suppression results in oxidative stress and dysfunction of mitochondrial and lipid metabolism, subsequently triggering tissue damage with many pathological consequences and clinical symptoms (16). It has been reported that estrogen promotes wound healing by ER $\beta$ independent of its anti-inflammatory activities, while the related mechanism remains unclear (17).

In an effort to investigate the potential mechanism for delayed wound healing in diabetes, we explored the role of oxidative stress and ER $\beta$ during hyperglycemia in endothelial cells (18). We found that transient hyperglycemia exposure induces persistent oxidative stress with maintained suppression of $\operatorname{ER} \beta$, SOD2, and the VEGF (vascular endothelial growth factor) signaling pathway after switching to normoglycemia. Further investigation showed that hyperglycemia-induced oxidative stress down-regulates ER $\beta$ expression, and subsequently suppresses SOD2 expression with a positive-feed forward loop to maintain elevated ROS generation even in subsequent normoglycemia. Either ER $\beta$ expression or ER $\beta$ agonist DPN breaks this loop for ROS generation and diminishes hyperglycemia-induced oxidative stress. The further in vivo diabetic rat model showed that $\mathrm{ER} \beta$ agonist DPN or SOD mimetic MnTBAP $(19,20)$ accelerates streptozotocin (STZ)induced delayed diabetic wound healing, while ER $\beta$ antagonist PHTPP mimicked the diabetic effect in non-diabetic rats. We conclude that ER $\beta$ accelerates diabetic wound healing by ameliorating hyperglycemia-induced persistent oxidative stress.

\footnotetext{
Abbreviations: ChIP, chromatin Immunoprecipitation; DFU, Diabetic foot ulcer; DPN, 2,3-bis(4-Hydroxyphenyl)-propionitrile; ER $\beta$, estrogen receptor $\beta$; HIF1 $\alpha$, hypoxia-inducible factor $\alpha$; HUVEC, human umbilical vein endothelial cells; MnTBAP, Mn(III) 5,10,15,20-tetrakis(4-carboxylatophenyl) porphyrin; $\mathrm{O}_{2}-$, superoxide anions; OVX, ovariectomised; PHTPP, 2-Phenyl3-(4-hydroxyphenyl)-5,7-bis(trifluoromethyl)-pyrazolo[1,5-a]pyrimidine, 4-[2-Phenyl-5,7-bis(trifluoromethyl)pyrazolo[1,5-a]-pyrimidin-3-yl]phenol; PPT, 4,4', 4" -(4-Propyl-[1H]-pyrazole-1,3,5-triyl; qPCR, quantitative real-time PCR; ROS, reactive oxygen species; SOD2, mitochondrial superoxide dismutase; STZ, streptozotocin; VEGF, vascular endothelial growth factor.
}

\section{MATERIALS AND METHODS}

\section{Materials and Reagents}

Primary Human Umbilical Vein Endothelial Cells (HUVECs, \# CC-2935, obtained Lonza) were maintained in EGMTM-Plus Media (from Lonza) with all the supplements in addition to charcoal-stripped Fetal Bovine Serum (\#12676029, Life Technologies) to remove traces of interfering basal estrogen. In some experiments, the HUVECs were conditionally immortalized by hTERT lentivirus vector with an extended life span to achieve higher transfection efficiency and experimental stability $(21,22)$. All cells were maintained in a humidified incubator with $5 \% \mathrm{CO}_{2}$ at $37^{\circ} \mathrm{C}$. The Hypoxia condition was induced by incubating in $94 \% \mathrm{~N} 2,5 \% \mathrm{CO} 2$ and $1 \% \mathrm{O}_{2}$ for $24 \mathrm{~h}$.

Antibodies for $\beta$-actin (sc-47778), ER $\beta$ (sc-137381), HIF1 $\alpha$ (sc-13515), SOD2 (sc-30080), and VEGF (sc-7269) were obtained from Santa Cruz Biotechnology. The antibody for CD31 (ab24590) was obtained from Abcam. 3-nitrotyrosine (3-NT) was measured by 3-Nitrotyrosine ELISA Kit (ab116691 from Abcam), and the HIF1 $\alpha$ transcriptional activity was measured by HIF1 $\alpha$ Transcription Factor Assay Kit (ab113104 from Abcam) in $50 \mu \mathrm{l}$ nuclear extracts from treated cells per manufacturers' instructions. Nuclear extracts were prepared using the NE-PER Nuclear and Cytoplasmic Extraction Reagents Kit (Pierce Biotechnology). The mitochondrial fraction was isolated using a Pierce Mitochondria Isolation Kit (Pierce Biotechnology) according to manufacturers' instructions. The protein concentration was measured using the Coomassie Protein Assay Kit (Pierce Biotechnology) per manufacturers' instructions. Luciferase activity assay was carried out using the Dual-Luciferase ${ }^{\mathrm{TM}}$ Assay System (Promega) and the transfection efficiency was normalized using a cotransfected renilla plasmid (23).

$\mathrm{ER} \alpha$ agonist PPT (\#1426), ER $\beta$ agonist DPN (\#1494) and ER $\beta$ antagonist PHTPP (\#2662) were obtained from Tocris. Streptozotocin (STZ, \#18883-66-4), and MnTBAP, a cell-permeable superoxide dismutase (SOD) mimetic and peroxynitrite scavenger (\#475870), were obtained from Sigma.

\section{Construction of SOD2/VEGF Reporter Plasmid}

The human genomic DNA was prepared from HUVECs cells. In order to construct SOD2/VEGF reporter plasmids, the SOD2 gene promoter (Ensembl gene ID: SOD2 ENST00000337404) and VEGF gene promoter (Ensembl gene ID: VEGFA-201 ENST00000230480.10) were amplified by PCR and subcloned into the pGL3-basic vector (\# E1751, Promega) using restriction sites of Mlu I and Hind III with the following primers: SOD2 forward: $5^{\prime}$-gcgc-acgcgt- gaa tcc tgt gga ttc atc ctt-3' (Mlu I) and SOD2 reverse: $5^{\prime}$ - gtac- aagctt- ctg aag acg aga aag cac agc- $3^{\prime}$ (Hind III); VEGF forward: $5^{\prime}$-gcgc-acgcgt- ctg tga acc ttg gtg ggg gtc-3' (Mlu I) and VEGF reverse: $5^{\prime}$ - gtac- aagctt -ctc gag agg tca $\operatorname{cct}$ tcc $\operatorname{cgc}-3^{\prime}$ (Hind III). All the vectors were verified by sequencing, and detailed information on these plasmids is available upon request (23). 


\section{Generation of Human ER $\beta / S O D 2$ Expression Lentivirus}

The human CDNA for ER $\beta$ and SOD2 was obtained from Open Biosystems. The cDNA for either human ER $\beta$ or SOD2 was subcloned into the pLVX-Puro vector (from Clontech) with the restriction sites of Xhol and Xbal using the below primers: $\operatorname{ER} \beta$ forward primer: $5^{\prime}$ - gtac - ctcgag- atg gat ata aaa aac tca $\mathrm{cca}-3^{\prime}(\mathrm{Xho} 1)$ and ER $\beta$ reverse primer: $5^{\prime}$ - gtac - tctaga- tca ctg ctc cat cgt tgc ttc- $3^{\prime}$ (Xba1); SOD2 forward primer: $5^{\prime}$ - gtacctcgag- atg ttg agc cgg gca gtg tgc- $3^{\prime}$ (Xho1) and SOD2 reverse primer: $5^{\prime}$ - gtac- tctaga- tta ctt ttt gca agc cat gta-3' (Xba1). The ER/SOD2 or empty control (CTL) was expressed through Lenti- $\mathrm{X}^{\mathrm{TM}}$ Lentiviral Expression Systems (from Clontech) per manufacturers' instructions (16).

\section{Measurement of ROS Generation}

Treated cells were seeded in a 24-well plate and incubated with $10 \mu \mathrm{M}$ CM-H2DCFDA (Invitrogen) for $45 \mathrm{~min}$ at $37^{\circ} \mathrm{C}$, and then the intracellular formation of reactive oxygen species (ROS) was measured at excitation/emission wavelengths of 485/530 nm using a FLx800 microplate fluorescence reader (Bio-Tek). The data was normalized as arbitrary units $(23,24)$.

\section{RT Reaction and Real-Time Quantitative PCR}

Total RNA from treated cells was extracted using the RNeasy Micro Kit (Qiagen), and the RNA was reverse transcribed using an Omniscript RT kit (Qiagen). All the primers were designed using Primer 3 Plus software with the Tm at $60^{\circ} \mathrm{C}$, primer size of $21 \mathrm{bp}$, and the product length in the range of 140-160 bp (see Table S1). The primers were validated with the amplification efficiency in the range of 1.9-2.1, and the amplified products were confirmed with agarose gel. The real-time quantitative PCR was run on iCycler iQ (Bio-Rad) with the Quantitect SYBR green PCR kit (Qiagen). The PCR was performed by denaturing at $95^{\circ} \mathrm{C}$ for $8 \mathrm{~min}$, followed by 45 cycles of denaturation at $95^{\circ} \mathrm{C}$, annealing at $60^{\circ} \mathrm{C}$, and extension at $72^{\circ} \mathrm{C}$ for $10 \mathrm{~s}$, respectively. One microliter of each cDNA was used to measure target genes. The $\beta$-actin was used as the housekeeping gene for transcript normalization, and the mean values were used to calculate relative transcript levels with the ${ }^{\Delta \Delta}$ CT method per instructions from Qiagen. In brief, the amplified transcripts were quantified by the comparative threshold cycle method using $\beta$-actin as a normalizer. Fold changes in gene mRNA expression were calculated as $2^{-\Delta \Delta C T}$ with $\mathrm{CT}=$ threshold cycle, $\Delta \mathrm{CT}=\mathrm{CT}$ (target gene $)-\mathrm{CT}(\beta$-actin $)$, and the $\Delta \Delta \mathrm{CT}=\Delta \mathrm{CT}$ (experimental)- $\Delta \mathrm{CT}$ (reference) $(16,23)$.

\section{Western Blotting}

Cells were lysed in an ice-cold lysis buffer $(0.137 \mathrm{M} \mathrm{NaCl}$, $2 \mathrm{mM}$ EDTA, $10 \%$ glycerol, $1 \%$ NP-40, $20 \mathrm{mM}$ Tris base, $\mathrm{pH}$ 8.0) with protease inhibitor cocktail (Sigma). The proteins were separated in $10 \%$ SDS-PAGE and further transferred to the PVDF membrane. The membrane was incubated with appropriate antibodies, washed and incubated with HRP-labeled secondary antibodies, and then the blots were visualized using the ECL+plus Western Blotting Detection System (Amersham). The blots were quantitated by IMAGEQUANT, and final results were normalized by $\beta$-actin $(16,23)$.

\section{Luciferase Reporter Assay}

$1.0 \times 10^{5}$ of SNK-6 cells were seeded in a 6 -well plate with complete medium to grow until they reached $80 \%$ confluence. Cells were then cotransfected by $3 \mu \mathrm{g}$ of VEGF full length or deletion reporter constructs, together with $0.2 \mu \mathrm{g}$ of pRLCMV-Luc Renilla plasmid (from Promega). Then, cells were treated by either $5 \mathrm{mM}$ aspirin or empty control (CTL) for $24 \mathrm{~h}$. After treatment, the cells were harvested and the luciferase activity assays were carried out using the Dual-Luciferase ${ }^{\mathrm{TM}}$ Assay System (Promega), and the transfection efficiencies were normalized using a cotransfected Renilla plasmid according to manufacturers' instructions. The VEGF reporter activity from different treatments was calculated (23).

\section{Chromatin Immunoprecipitation (ChIP)}

Cells were washed and crosslinked using 1\% formaldehyde for $20 \mathrm{~min}$ and terminated by $0.1 \mathrm{M}$ glycine. Cell lysates were sonicated and centrifuged. Five hundred microgram of protein were pre-cleared by BSA/salmon sperm DNA with preimmune IgG and a slurry of Protein A Agarose beads. Immunoprecipitations were performed with the indicated antibodies, BSA/salmon sperm DNA and a 50\% slurry of Protein $\mathrm{A}$ agarose beads. Input and immunoprecipitates were washed and eluted, then incubated with $0.2 \mathrm{mg} / \mathrm{ml}$ Proteinase $\mathrm{K}$ for $2 \mathrm{~h}$ at $42^{\circ} \mathrm{C}$, followed by $6 \mathrm{~h}$ at $65^{\circ} \mathrm{C}$ to reverse the formaldehyde crosslinking. DNA fragments were recovered by phenol/chloroform extraction and ethanol precipitation. A $~ 150$ bp fragment on the SOD2 or VEGF promoter was amplified by real-time PCR (qPCR) using the primers provided in Table S1 (16, 23).

\section{SOD2 Activity Assay}

The SOD2 was obtained from the mitochondrial fraction that was isolated using a Pierce Mitochondria Isolation Kit (Pierce) according to manufacturers' instructions. SOD activity was measured as described previously (25). In brief, a stable $\mathrm{O} 2{ }^{-}$source was generated through the conversion action of XOD (xanthine oxidase) from xanthine and was mixed with chemiluminescent (CL) reagents to achieve a stable light emission. The SOD2 sample injection can scavenge $\mathrm{O}_{2}^{-}$ and the subsequent decrease of chemiluminescent response is proportional to the SOD2 activity. This system can have a detection limit of 0.001 U.ml-1 with the linear range of $0.03 \sim 2.00$ U.ml-1. The results were normalized by protein concentration and expressed as Units/mg proteins (U/mg) (15).

\section{In vivo Rat Experiments}

The animal protocol conformed to US NIH guidelines (Guide for the Care and Use of Laboratory Animals, No. 85-23, revised 1996), and was reviewed and approved by the Institutional Animal Care and Use Committee from Wuhan University. The female rats were housed 4 or 5 per cage on a 12:12-h lightdark cycle and were given phytoestrogen-free commercial rodent chow and water ad libitum on arrival. Estrous cycles were 
monitored with daily vaginal smears. Only rats with at least two regular 4 to $5 \mathrm{~d}$ estrous cycles were included in the studies. 6week old females were anesthetized using a ketamine/xylazine mixture (80 and $4 \mathrm{mg} / \mathrm{kg}$, respectively, intramuscular), and ovariectomized (OVX), then the OVX rats were ready for further experiments after 1-week recovery (26).

\section{Diabetic Rat Model}

Chronic diabetic rats were induced by injection of $50 \mathrm{mg} / \mathrm{kg}$ streptozotocin (STZ, 0.05 M sodium citrate, $\mathrm{pH} 5.5$ ) after an $8 \mathrm{~h}$ fasting. Animals with blood glucose $>300 \mathrm{mg} / \mathrm{dl}$ were considered positive, while control (CTL) rats received only vehicle injection.

\section{Rat Models of Cutaneous Burn}

Wild type and diabetic rats were subjected to a model of cutaneous burn injury. The dorsum of each rat was shaved with electric clippers and depilated with Nair. The rats were anesthetized by inhalation of $5 \%$ isoflurane, and then the cutaneous burn injury was made on the dorsa of the rats by exposure to a hot copper pillar $(2-\mathrm{cm}$ diameter $)$ at $75^{\circ} \mathrm{C}$ for $15 \mathrm{~s}$.

\section{Experimental Groups}

The experimental rats were separated into 5 groups: Group 1 . Wild type (CTL) rats received only subcutaneously vehicle (5\% DMSO in maize oil) injection; Group 2. Diabetic (STZ) rats received only vehicle injection; Group 3. Diabetic (STZ) rats received $450 \mu \mathrm{g} / \mathrm{kg} /$ day of DPN (dissolved in DMSO) injection on days of $0,4,8,12$, and 16 with respect to the time of wounding (STZ/DPN); Group 4. STZ rats received $10 \mathrm{mg} / \mathrm{kg} /$ day of MnTBAP (dissolved in DMSO) injection (STZ/MnTBAP); Group 5. CTL rats received $450 \mu \mathrm{g} / \mathrm{kg} /$ day of PHTPP (dissolved in DMSO) injection (CTL/PHTPP).

\section{Measurement}

Digital photographs of the wounds were taken every 2 days for 20 days. Wound area was quantified as a percent area of the original wound size using Image $J$ software. At indicated time points, wounds were excised and snap-frozen or, alternatively, processed for H\&E staining. Vascular density was detected on frozen sections by immunohistochemistry using CD31 mouse monoclonal antibody. For quantification of CD31 positivity, wounds were analyzed under $200^{x}$ magnification, and the number of positive 6 cells per high-power field (HPF) were counted. All counts and observations were performed by a blinded observer (27).

\section{Immunohistochemistry}

The tissues were dissected and snap-frozen in the OCT compound. The $10 \mu \mathrm{m}$ sections were cut by clean microtome and mounted on PEN-membrane slides $(2.0 \mu \mathrm{m}$, Leica), and stored in $-20^{\circ} \mathrm{C}$ before use. The slides were first fixed by $3.7 \%$ formaldehyde at $37^{\circ} \mathrm{C}$ for $15 \mathrm{~min}$, then permeabilized by $1 \% \mathrm{BSA}+0.2 \%$ Triton $\mathrm{X}-100$ in PBS for $1 \mathrm{~h}$, then blotted with $40 \mu \mathrm{g} / \mathrm{ml}$ (dilute 1:20) of either VEGF or CD31 mouse monoclonal antibody for $2 \mathrm{~h}$, washed three times and the Texasred labeled anti-mouse secondary antibody (1:200) was added for blotting for another $1 \mathrm{~h}$. After thorough washing, the slides were visualized and photographed. The relative densities of each group were quantitated for protein expression using Image J. software (22).

\section{In vivo Superoxide Release}

Superoxide anion $\left(\mathrm{O}_{2}{ }^{-}\right)$release from the tissue was determined by a luminol-EDTA-Fe enhanced chemiluminescence (CL) system supplemented with DMSO-TBAC (Dimethyl sulfoxidetetrabutyl-ammonium chloride) solution for extraction of released $\mathrm{O}_{2}$ - from tissues as described previously (24). The superoxide levels were calculated from the standard curve generated by the xanthine/xanthine oxidase reaction (23).

\section{Statistical Analysis}

The data was given as mean \pm SEM; all of the experiments were performed at least in quadruplicate unless otherwise indicated. The one-way ANOVA followed by the Bonferroni post hoc test was used to determine statistical significance of different groups. The mouse survival curve was determined by Kaplan-Meier survival analysis using SPSS 22 software and a $P$-value $<0.05$ was considered significant (23).

\section{RESULTS}

\section{ER $\beta$ Expression Diminishes Hyperglycemia-Induced Persistent ROS Generation}

We first measured the effect of hyperglycemia on ROS generation in the HUVECs. The HUVECs were firstly exposed to high glucose $(25 \mathrm{mM})$ for 4 days, then switched to low glucose ( $5 \mathrm{mM})$ for additional 4 days. We found that ROS generation slightly increased, continuously increasing to $187 \%$ on day 3 , and then significantly increasing to $276 \%$ on day 4 compared to day 0 . On day 5 , the ROS generation slightly decreased, but maintained high levels with a $220 \%$ increase. These high levels of ROS lasted for 4 days with a $207 \%$ increase (on day 8) compared to day 0 . On the other hand, the elevated ROS generation completely diminished after infection of either ER $\beta$ lentivirus ( $\uparrow \mathrm{ER} \beta$ ), or SOD2 lentivirus ( $\uparrow \mathrm{SOD} 2)$, or treated by $100 \mu \mathrm{M}$ ER $\beta$ agonist (DPN) on day 4 (see Figure 1A). We also evaluated the potential effect of $\mathrm{ER} \alpha$ agonist PPT, and found that $100 \mu \mathrm{M}$ PPT treatment on day 4 (PPT(d4)) had no effect on ROS generation (see Figure S1). We then measured the 3nitrotyrosine (3-NT) generation. The results showed that 3-NT generation remains high after 4-day hyperglycemia treatment followed by 4-day normoglycemia ( $\mathrm{HG}(4 \mathrm{~d})+\mathrm{LG}(4 \mathrm{~d})$ ) compared to the 8-day normoglycemia treatment $(\mathrm{LG}(8 \mathrm{~d}))$ group. Again, infection of either ER $\beta$ lentivirus $(\mathrm{HG}(4 \mathrm{~d})+\mathrm{LG}(4 \mathrm{~d}) / \uparrow \mathrm{ER} \beta)$, or SOD2 lentivirus (HG(4d)+LG(4d)/个SOD2), or treatment with $100 \mu \mathrm{M}$ ER $\beta$ agonist $(\mathrm{HG}(4 \mathrm{~d})+\mathrm{LG}(4 \mathrm{~d}) / \uparrow \mathrm{DPN})$ on day 4 completely restored 3-NT generation to normal (see Figure 1B). Our results indicate that expression of either ER $\beta$ or SOD2, or ER $\beta$ agonist treatment, diminishes hyperglycemia-induced persistent ROS generation. 

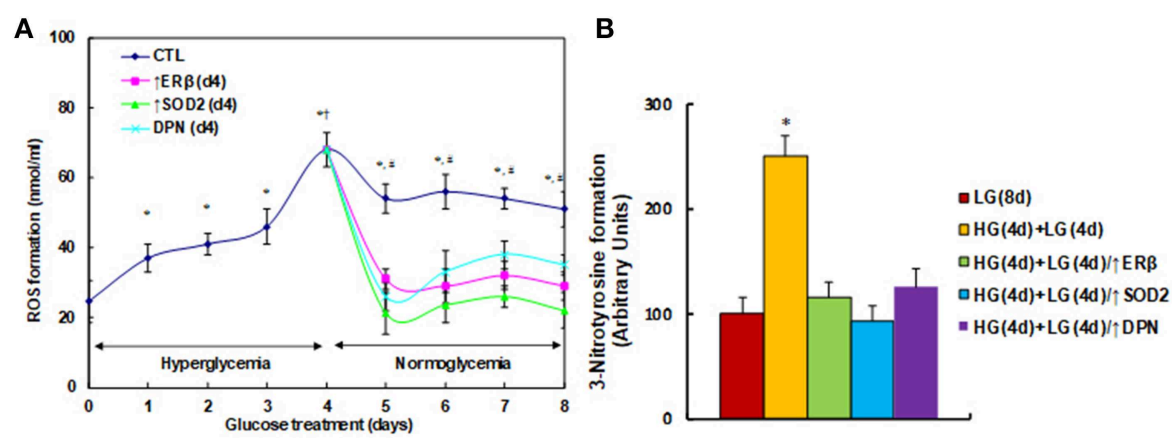

FIGURE 1 | ER $\beta$ expression diminishes hyperglycemia-induced persistent ROS generation. (A) HUVECs were incubated in hyperglycemia (25 mM glucose) for 4 days, and then switched to normoglycemia ( $5 \mathrm{mM}$ glucose) for an additional 4 days. On day 4 , cells were infected by an empty vector (CTL), ER $\beta$ lentivirus (ER $\beta \uparrow)$, or SOD2 lentivirus (SOD2 $\uparrow$ ), or treated by $100 \mu \mathrm{M} E R \beta$ agonist (DPN) for a subsequent 4 days. The cells were harvested each day for analysis of ROS formation, $n=4$. ${ }^{\star} P<0.05$, vs. day 0 group; ${ }^{\dagger} P<0.05$, vs. day 3 group; $\# P<0.05$, vs. day 4 group. (B) The HUVECs cells were treated either in normoglycemia (5mM) for 8 days $(\mathrm{LG}(8 \mathrm{~d})$ ), or in hyperglycemia ( $\mathrm{HG}$ in $25 \mathrm{mM}$ glucose) for 4 days followed by normoglycemia (LG in $5 \mathrm{mM}$ glucose) for an additional 4 days (HG(4d)+LG(4d)), or the cells were infected at day 4 by either ER $\beta$ lentivirus $(H G(4 d)+L G(4 d) / E R \beta \uparrow)$, or SOD2 lentivirus $(H G(4 d)+L G(4 d) / S O D 2 \uparrow)$, or treated by ER $\beta$ agonist $(H G(4 d)+L G(4 d) / D P N)$ for subsequent 4 days, and the cells were harvested for the analysis of 3-nitrotyrosine formation, $n=5$. ${ }^{\star} P<0.05$, vs. $L G(8 d)$ group. Data are expressed as mean \pm SEM.

\section{ER $\beta$ Expression Diminishes Hyperglycemia-Induced Persistent SOD2 Suppression}

We measured the effect of hyperglycemia on gene expression in HUVECs. The HUVECs were firstly exposed to high glucose $(25 \mathrm{mM})$ for 4 days, then switched to low glucose $(5 \mathrm{mM})$ for an additional 4 days as shown in Figure 1. We then measured the mRNA expression of ER $\beta$ (see Figure 2A) and SOD2 (see Figure 2B). The results showed that $\operatorname{ER} \beta$ expression slightly increased to $126 \%$ on day 2 , and then started to decrease on day 3. It decreased to $56 \%$ on day 4 , and after switching from hyperglycemia to normoglycemia on days $5,6,7$ and 8 , the expression of both ER $\beta$ and SOD2 remained as low as on day 4. Infection of $\operatorname{ER} \beta$ lentivirus $(\uparrow E R \beta)$ on day 4 significantly increased expression of ER $\beta$ and SOD2, and the expression remained high throughout the next 4 days. On the other hand, either infection of SOD2 lentivirus ( $\uparrow$ SOD2), or ER $\beta$ agonist (DPN) treatment significantly increased SOD2 expression, but did not increase ER $\beta$ expression. We also evaluated the potential effect of ER $\alpha$ agonist PPT, and found that $100 \mu \mathrm{M}$ PPT treatment on day 4 (PPT(d4)) had no effect on the expression of either ER $\beta$ or SOD2 (see Figure S2). These results indicate that ER $\beta$ may be the upstream target gene of SOD2. We then measured the binding ability of ER $\beta$ on the SOD 2 promoter by ChIP analysis (see Figure 2C). It showed that ER $\beta$ binding ability decreased to $58 \%$ as a result of $\mathrm{HG}(4 \mathrm{~d})+\mathrm{LG}(4 \mathrm{~d})$ treatment compared to the $L G(8 d))$ group, and this was completely restored by either infection of $\operatorname{ER} \beta$ lentivirus ( $\uparrow \mathrm{ER} \beta$ ) or SOD2 lentivirus ( $\uparrow \mathrm{SOD} 2$ ), or $\operatorname{ER} \beta$ agonist (DPN) treatment. We also measured SOD2 luciferase reporter activity (see Figure 2D). We found that $\mathrm{ER} \beta$ binding ability decreased to $58 \%$ as a result of $\mathrm{HG}(4 \mathrm{~d})+\mathrm{LG}(4 \mathrm{~d})$ treatment compared to LG(8d) group, and this was completely restored by SOD2 lentivirus infection ( $\uparrow$ SOD2). The infection of $\mathrm{ER} \beta$ lentivirus $(\uparrow \mathrm{ER} \beta)$ or $\mathrm{ER} \beta$ agonist (DPN) treatment further increased SOD2 reporter activity to 156 and $135 \%$, respectively. We also measured the protein expression for ER $\beta$ and SOD2 (see
Figures 2E,F). The results showed that $\operatorname{ER} \beta$ protein decreased to $64 \%$ as a result of $\mathrm{HG}(4 \mathrm{~d})+\mathrm{LG}(4 \mathrm{~d})$ treatment compared to the $L G(8 d)$ group, and infection of $\operatorname{ER} \beta$ lentivirus ( $\uparrow E R \beta)$ increased ER $\beta$ expression to $216 \%$, while either SOD2 lentivirus infection ( $\uparrow$ SOD2), or ER $\beta$ agonist (DPN) treatment showed no effect. On the other hand, SOD2 protein decreased to $51 \%$ as a result of $\mathrm{HG}(4 \mathrm{~d})+\mathrm{LG}(4 \mathrm{~d})$ treatment compared to the $\mathrm{LG}(8 \mathrm{~d})$ group, and the lentivirus infection of either $\operatorname{ER} \beta(\uparrow \operatorname{ER} \beta)$ or SOD2 ( $\uparrow$ SOD2), or ER $\beta$ agonist (DPN) treatment, increased SOD2 protein level by 169,194 , and $176 \%$, respectively. Finally, we measured the SOD2 enzyme activity (see Figure 2G). The results showed that the activity decreased to $51 \%$ as a result of HG(4d)+LG(4d) treatment compared to the LG(8d) group, and the lentivirus infection of either $\operatorname{ER} \beta(\uparrow \mathrm{ER} \beta)$, SOD2 ( $\uparrow$ SOD2), or $\mathrm{ER} \beta$ agonist (DPN) treatment increased SOD2 enzyme activity by 137,124 , and $142 \%$, respectively. Our results indicate that SOD2 expression is regulated by $\operatorname{ER} \beta$, and $\operatorname{ER} \beta$ overexpression by lentivirus or ER $\beta$ activation by $\operatorname{ER} \beta$ agonist DPN upregulates SOD2 expression and subsequently diminishes hyperglycemiainduced persistent ROS generation.

\section{ER $\beta$ Expression Diminishes Hyperglycemia-Induced Persistent Suppression of the HIF $1 \alpha /$ VEGF Signaling Pathway}

We measured the effect of hyperglycemia on the HIF1 $\alpha /$ VEGF signaling pathway. The HUVECs were cultured in hypoxia conditions and were treated first in high glucose $(25 \mathrm{mM})$ for 4 days, and then switched to low glucose $(5 \mathrm{mM})$ for an additional 4 days as described in Figure 1B. We first measured the mRNA expression levels for HIF1 $\alpha$ and VEGF (see Figure 3A). The results showed that HIF1 $\alpha$ mRNA levels have no significant changes in different treatments, while the VEGF mRNA levels decreased to $39 \%$ as a result of $\mathrm{HG}(4 \mathrm{~d})+\mathrm{LG}(4 \mathrm{~d})$ treatment, and the infection of either $\operatorname{ER} \beta$ lentivirus $(\uparrow E R \beta)$ or SOD2 lentivirus ( $\uparrow$ SOD2), or ER $\beta$ agonist DPN increased VEGF mRNA to 136, 

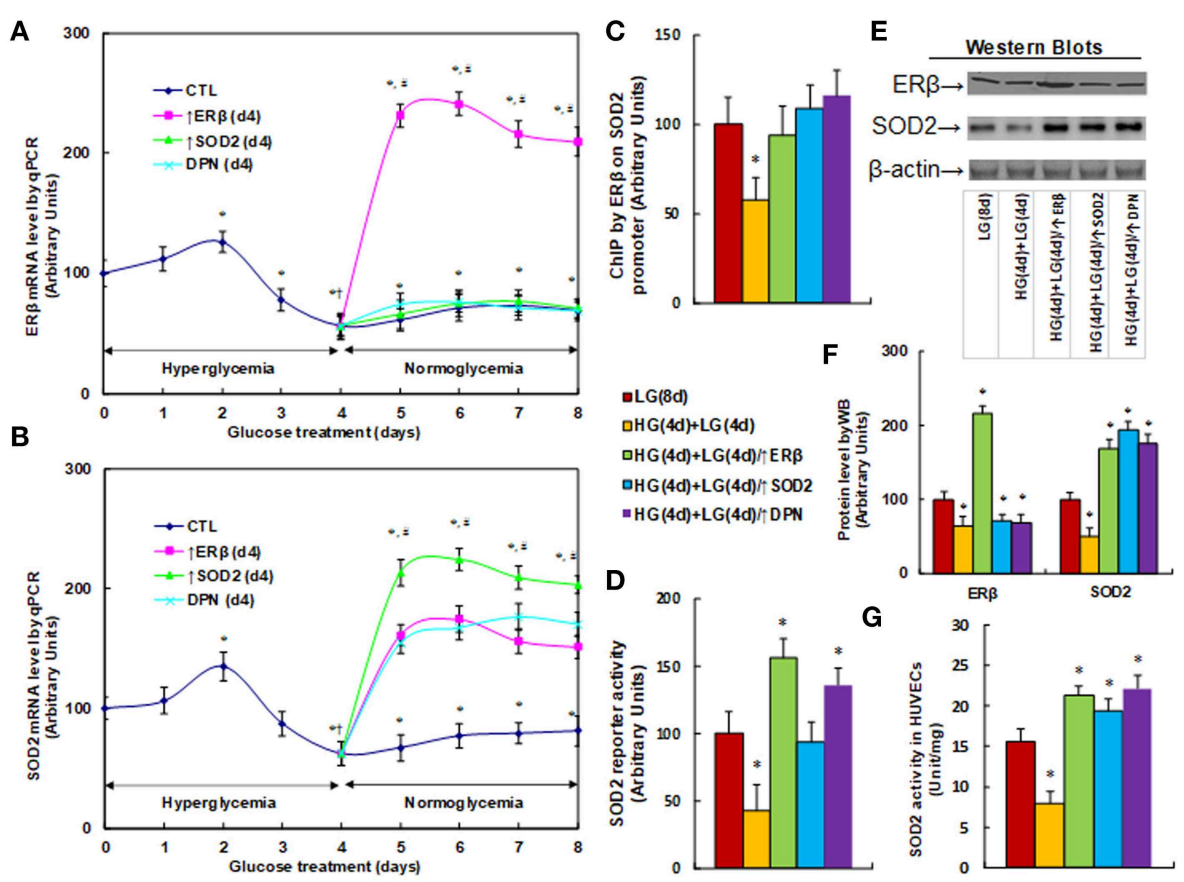

FIGURE 2 | ER $\beta$ expression diminishes hyperglycemia-induced persistent SOD2 suppression. (A,B) HUVECs were incubated in hyperglycemia (25 mM glucose) for 4 days, and then switched to normoglycemia ( $5 \mathrm{mM}$ glucose) for an additional 4 days. On day 4 , cells were either infected by empty vector (CTL), ER $\beta$ lentivirus (ER $\beta \uparrow)$, or SOD2 lentivirus (SOD2 $\uparrow$ ), or treated by $100 \mu \mathrm{M}$ ER $\beta$ agonist (DPN) for a subsequent 4 days, and the cells were harvested for mRNA analysis of ER $\beta$ (A) and SOD2 (B), $n=4$. ${ }^{\star} P<0.05$, vs. day 0 group; ${ }^{\dagger} P<0.05$, vs. day 3 group; $\# P<0.05$, vs. day 4 group. (C-G) The HUVECs cells were treated either in normoglycemia $(5 \mathrm{mM})$ for 8 days $(\mathrm{LG}(8 \mathrm{~d}))$, or in hyperglycemia ( $\mathrm{HG}$ in $25 \mathrm{mM}$ glucose) for 4 days followed by normoglycemia ( $\mathrm{LG}$ in $5 \mathrm{mM}$ glucose) for an additional 4 days $(\mathrm{HG}(4 \mathrm{~d})+\mathrm{LG}(4 \mathrm{~d}))$, or the cells were infected at day 4 by either $E R \beta$ lentivirus $(\mathrm{HG}(4 \mathrm{~d})+\mathrm{LG}(4 \mathrm{~d}) / \mathrm{ER} \beta \uparrow)$, or SOD2 lentivirus $(\mathrm{HG}(4 \mathrm{~d})+\mathrm{LG}(4 \mathrm{~d}) / \mathrm{SOD} 2 \uparrow)$, or treated by $E R \beta$ agonist $(\mathrm{HG}(4 \mathrm{~d})+\mathrm{LG}(4 \mathrm{~d}) / \mathrm{DPN})$ for a subsequent 4 days, and the cells were harvested for further analysis. (C) ChIP analysis by ER $\beta$ antibody on SOD2 promoter, $n=4$. (D) SOD2 reporter activity assay, $n=5$. (E) Representative picture for western blots. (F) Protein quantitation for (E), $n=4$. (G) SOD2 activity assay, $n=5$. ${ }^{*} P<0.05$, vs. LG(8d) group. Data are expressed as mean \pm SEM.

162 , and $115 \%$, respectively compared to the $L G(8 \mathrm{~d})$ group. We then measured protein expression through western blotting (see Figures 3B,C). The results showed that HIF1 $\alpha$ protein levels have no significant changes under different treatments, while the VEGF protein level decreased to $41 \%$ as a result of HG(4d)+LG(4d) treatment, and the infection of either ER $\beta$ lentivirus $(\uparrow \mathrm{ER} \beta)$ or SOD2 lentivirus ( $\uparrow \mathrm{SOD} 2)$, or treatment of ER $\beta$ agonist DPN, increased VEGF protein to 143,187 , and $113 \%$, respectively compared to the $L G(8 d)$ group. We then measured the HIF1 $\alpha$ transcriptional activity (see Figure 3D). The results showed that HIF $1 \alpha$ transcriptional activity decreased to $53 \%$ as a result of $\mathrm{HG}(4 \mathrm{~d})+\mathrm{LG}(4 \mathrm{~d})$ treatment compared to the $L G(8 d)$ group, and the infection of either ER $\beta$ lentivirus $(\uparrow \mathrm{ER} \beta$ ) or SOD2 lentivirus ( $\uparrow \mathrm{SOD} 2)$, or ER $\beta$ agonist DPN treatment, completely restored this effect. We also measured the HIF1 $\alpha$ binding ability on the VEGF promoter by ChIP analysis (see Figure 3E). It showed that HIF1 $\beta$ binding ability was decreased to $36 \%$ by $\mathrm{HG}(4 \mathrm{~d})+\mathrm{LG}(4 \mathrm{~d})$ treatment compared to $L G(8 d)$ ) group, and this was completely restored by either infection of $E R \beta$ lentivirus ( $\uparrow E R \beta$ ) or SOD2 lentivirus ( $\uparrow S O D 2$ ), or $\mathrm{ER} \beta$ agonist (DPN) treatment. Finally, we measured the VEGF luciferase reporter activity (see Figure 3F). The results showed that VEGF reporter activity decreased to $56 \%$ as a result of $\mathrm{HG}(4 \mathrm{~d})+\mathrm{LG}(4 \mathrm{~d})$ treatment, and the infection of either ER $\beta$ lentivirus $(\uparrow E R \beta)$ or SOD2 lentivirus ( $\uparrow$ SOD2), or $\mathrm{ER} \beta$ agonist DPN, increased VEGF reporter activity to 139 , 136 , and $115 \%$, respectively compared to the $L G(8 \mathrm{~d})$ group. The results indicated that VEGF expression is regulated by HIF $1 \alpha$ under hypoxia conditions, and the expression of either $\mathrm{ER} \beta$ or SOD2, or ER $\beta$ agonist DPN treatment, diminishes hyperglycemia-induced persistent ROS generation, activates HIF1 $\alpha$ transcriptional activity, and subsequently upregulates VEGF expression, favoring wound healing.

\section{ER $\beta$ Activation Restores Diabetes-Induced Persistent Oxidative Stress and VEGF Suppression}

We evaluate the potential effect of ER $\beta$ activation on oxidative stress and the VEGF signaling pathway in diabetic wound healing in rats. The burn injury was introduced in either control (CTL) or diabetic (STZ) rats, and then treated with either ER $\beta$ agonist DPN (STZ/DPN), or SOD mimetic MnTBAP (STZ/MnTBAP), or ER $\beta$ antagonist PHTPP (CTL/PHTPP), and the wound tissues were collected for the further analysis. We first measured the superoxide anion release from the wound tissues (see Figure 4A). The results showed that superoxide anion $\left(\mathrm{O}_{2}{ }^{-}\right)$release increased to $214 \%$ in the STZ group compared to 

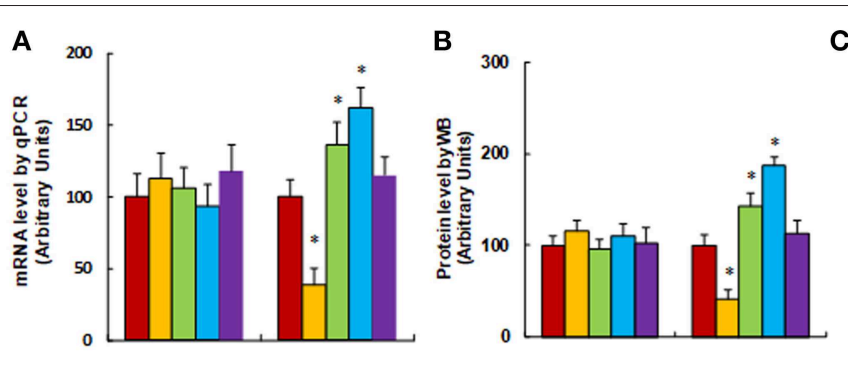

D

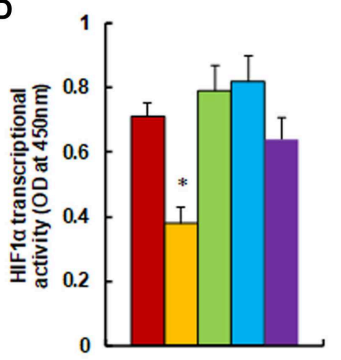

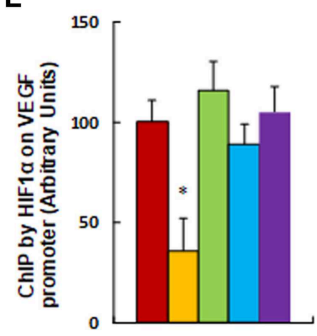

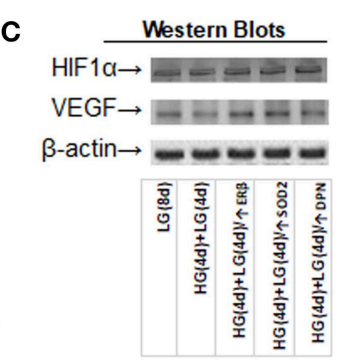

F

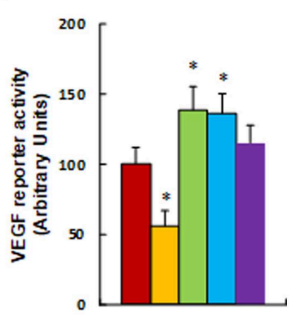

FIGURE 3 | ER $\beta$ expression diminishes hyperglycemia-induced persistent suppression of the HIF1 $\alpha$ NEGF signaling pathway. The HUVECs cells were cultured in hypoxia conditions and treated either in normoglycemia $(5 \mathrm{mM})$ for 8 days (LG(8d)), or in hyperglycemia ( $\mathrm{HG}$ in $25 \mathrm{mM}$ glucose) for 4 days followed by normoglycemia (LG in $5 \mathrm{mM}$ glucose) for an additional 4 days (HG(4d)+LG(4d)), or the cells were infected at day 4 by either $E R \beta$ lentivirus $(H G(4 d)+L G(4 d) / E R \beta \uparrow)$, or SOD2 lentivirus $(\mathrm{HG}(4 \mathrm{~d})+\mathrm{LG}(4 \mathrm{~d}) / \mathrm{SOD} 2 \uparrow)$, or treated by $E R \beta$ agonist $(\mathrm{HG}(4 \mathrm{~d})+\mathrm{LG}(4 \mathrm{~d}) / \mathrm{DPN})$ for subsequent 4 days, and the cells were harvested for further analysis. (A) mRNA levels by qPCR, $n=4$. (B) Protein quantitation for western blots, $n=4$. (C) Representative pictures for (B). (D) HIF1 $\alpha$ transcriptional activity assay, $n=5$. (E) ChIP analysis by HIF1 $\alpha$ antibody on VEGF promoter, $n=4$. (F) VEGF reporter activity assay, $n=5$. ${ }^{*}<<0.05$, vs. LG(8d) group. Data are expressed as mean \pm SEM.

the CTL group. Either DPN (STZ/DPN) or MnTBAP treatment (STZ/MnTBAP) in STZ rats completely restored this effect, while PHTPP treatment in CTL rats (CTL/PHTPP) increased superoxide anion release to $227 \%$ compared to the CTL group, mimicking the effect of STZ rats. We also measured the mRNA expression of ER $\beta$, SOD2 and VEGF (see Figure 4B). The results showed that ER $\beta$ expression was decreased to $46 \%$ in the STZ group compared to the CTL group, and both ER $\beta$ agonist DPN and antagonist PHTPP showed no effect on ER $\beta$ expression, while SOD mimetic MnTBAP (STZ/MnTBAP) completely restored the effect of STZ. In addition, the mRNA expression of SOD2 and VEGF decreased to 53 and $42 \%$, respectively, in STZ treatment, and this effect was completely restored by DPN (STZ/DPN) and MnTBAP (STZ/MnTBAP) treatment. On the other hand, the mRNA expression of SOD2 and VEGF decreased to 69 and 57\%, respectively, in the PHTPP treatment (CTL/PHTPP), mimicking the effect of STZ treatment. We then measured the protein expression, and a pattern similar to that of mRNA expression was observed for the protein levels of ER $\beta$, SOD2 and VEGF (see Figures 4C,D). We also evaluated VEGF expression using immunohistochemistry (see Figure 4E), and the results showed that the VEGF staining in wound tissues was consistent with the results of the western blots as shown in Figure 4C. Finally, we measured the SOD2 enzyme activity in wound tissues (see Figure 4F). The results showed that SOD2 activity was decreased to $53 \%$ in STZ group compared to CTL group, and this effect was completely restored by DPN (STZ/DPN) and MnTBAP (STZ/MnTBAP) treatment, and the PHTPP treatment (CTL/PHTPP) mimicked the effect of STZ group. The results indicate that $\mathrm{ER} \beta$ activation restores diabetesinduced persistent oxidative stress and VEGF suppression.

\section{ER $\beta$ Activation Accelerates Wound Healing in Diabetic Rats}

We evaluated the potential effect of ER $\beta$ activation on wound healing in rats with burn injuries. The burn injury was introduced in either control (CTL) or diabetic (STZ) rats, and then treated with ER $\beta$ agonist DPN (STZ/DPN), SOD mimetic MnTBAP (STZ/MnTBAP), or ER $\beta$ antagonist PHTPP (CTL/PHTPP), and rate of wound healing was evaluated. We first evaluated the wound healing rate in different treatments. In Figure $\mathbf{5 A}$, the representative pictures for wound area were taken on day 12, and the results showed that STZ rats had significantly delayed wound healing compared to CTL group, and the treatments of DPN (STZ/DPN) and MnTBAP (STZ/MnTBAP) completely restored this effect, while the PHTPP treatment in control rats (CTL/PHTPP) mimicked the effect of STZ rats. In Figure 5B, the relative wound areas were quantitated following different time points, and the results showed that STZ rats and CTL/PHTPP group had significantly delayed wound healing compared to groups of CTL, CTL/DPN and CTL/MnTBAP. We then evaluated the granulation tissue deposition by $H \& E$ staining on day 12 after the introduction of the burn injury (see Figure 5C). The results showed that the STZ group had significant less granulation tissue deposition compared to the CTL group. Both DPN (STZ/DPN) and MnTBAP (STZ/MnTBAP) treatments in STZ rats restored this effect, while PHTPP in CTL rats (CTL/PHTPP) mimicked the effect of STZ rats. Finally, we 


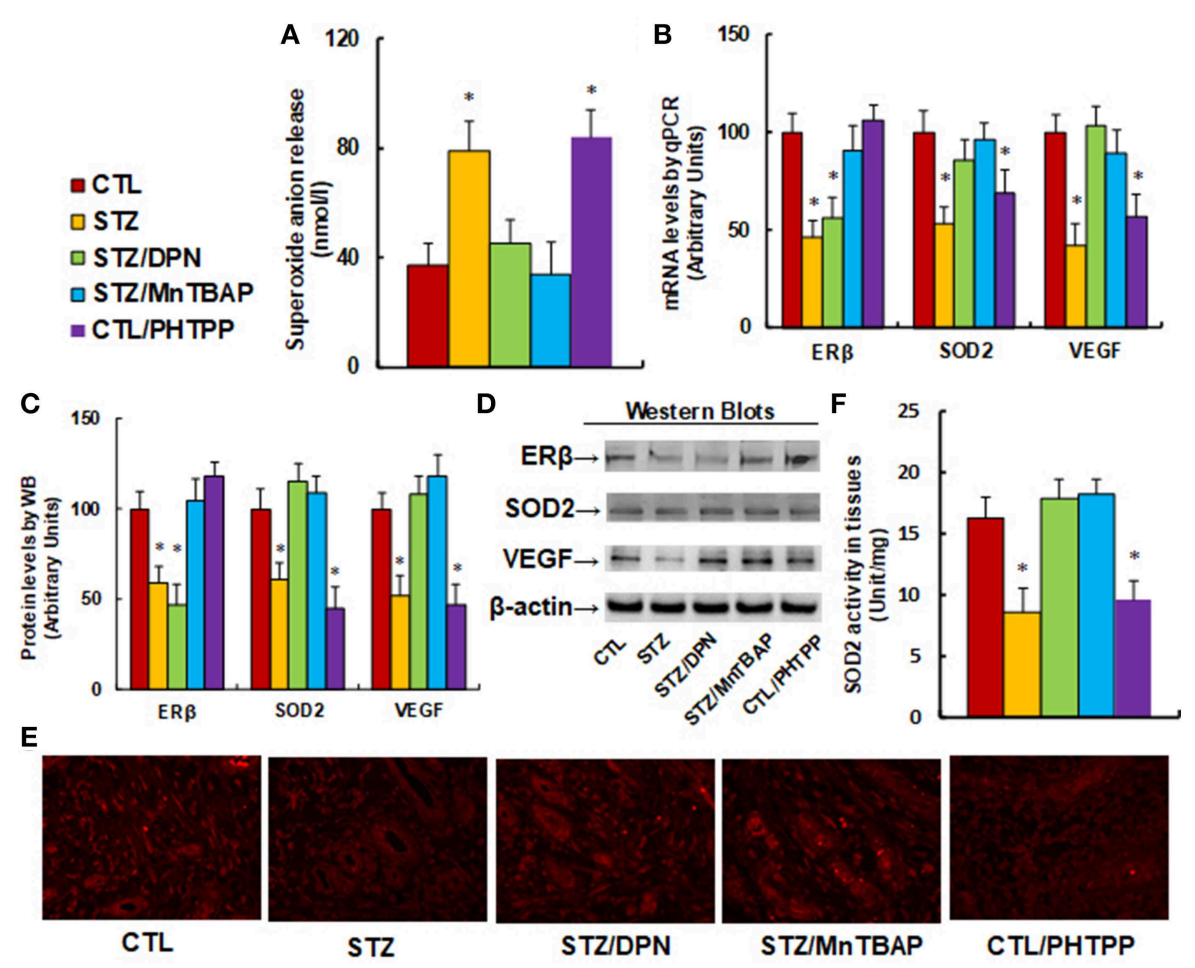

FIGURE 4 | ER $\beta$ activation restores diabetes-induced persistent oxidative stress and VEGF suppression. The wounds were excised and collected from the experimental rats with either control rats (CTL), or STZ diabetic rats (STZ), or STZ rats treated by $450 \mu \mathrm{g} / \mathrm{kg} / \mathrm{day}$ of DPN (STZ/DPN), or STZ rats treated by 10 $\mathrm{mg} / \mathrm{kg} /$ day of MnTBAP (STZ/MnTBAP), or CTL rats treated by $450 \mu \mathrm{g} / \mathrm{kg} /$ day of PHTPP (CTL/PHTPP) for further analysis. (A) Superoxide anion release, $n=5$. (B) mRNA levels by qPCR, $n=4$. (C) Protein quantitation for western blots, $n=4$. (D) Representative pictures for (C). (E) Representative pictures for VEGF expression by immunohistochemistry. (F) SOD2 activity assay, $n=5$. ${ }^{*} P<0.05$, vs. CTL group. Data are expressed as mean \pm SEM.

measured neovascularization by evaluating $\mathrm{CD}^{+} 1^{+}$positive $\left(\mathrm{CD} 31^{+}\right)$cells using CD31 immunohistochemistry staining (see Figures 5D,E). In Figure 5D, the CD $31^{+}$cells were decreased to 23,48 , and $26 \%$, respectively on days 6, 12 and 18 in STZ rats compared to CTL rats; this effect was completely restored by treatments of DPN (STZ/DPN) and MnTBAP (STZ/MnTBAP), while the PHTPP treatment in CTL rats (CTL/PHTPP) mimicked the effect of STZ rats. In addition, the representative pictures of CD31 staining were taken for the wound tissues on day 12 (see Figure 5E), the results showed the consistency with the quantitative details as shown in Figure 5D. Our results indicate that $\operatorname{ER} \beta$ activation accelerates wound healing in diabetic rats.

\section{DISCUSSION}

In this study, we demonstrated that transient hyperglycemia exposure induces maintained ROS generation, resulting in suppression of ER $\beta$ and SOD2 and forming a positive feedforward loop for ROS generation in subsequent normoglycemia. $\operatorname{ER} \beta$ expression breaks this loop and restores hyperglycemiainduced oxidative stress. Furthermore, in vivo diabetic rat models showed that ER $\beta$ agonist DPN treatment ameliorates hyperglycemia-induced ROS generation and accelerates diabetic wound healing. This provides a new strategy for the clinical treatment of diabetic wounds based on $\operatorname{ER} \beta$ activation.

\section{Hyperglycemia-Induced Positive Feed-Forward Loop for ROS Generation}

Our results showed that hyperglycemia-induced ROS generation suppresses ER $\beta$ expression, and subsequently suppresses its downstream target gene SOD2 (12). The SOD2 suppression then results in additional ROS over-generation. This generates a positive feed-forward loop for ROS generation of ROS, triggering several pathophysiological signaling pathways. This effect remains active after the removal of the original driving force by the switch from hyperglycemia to normoglycemia. This partly explains the potential mechanism of the hyperglycemia memory $(8,9)$, and our findings show that ER $\beta$ expression or activation (28) may be able to break this kind of loop, and subsequently diminish the hyperglycemia memory.

\section{Hyperglycemia-Induced ER $\beta$ Suppression}

Our results showed that transient hyperglycemia exposure induces persistent ER $\beta$ suppression after switching to normoglycemia. This suggests that $\operatorname{ER} \beta$ may play an important role in hyperglycemia-induced diabetic complications and delayed diabetic wound healing $(29,30)$, although the detailed 
A

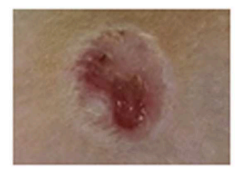

CTL

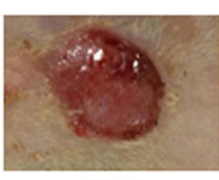

STZ

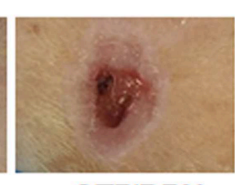

STZIDPN

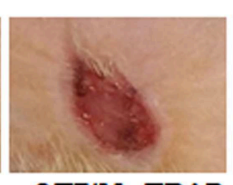

STZ/MnTBAP

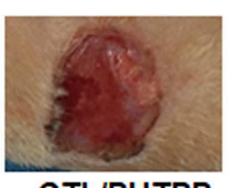

CTL/PHTPP

B

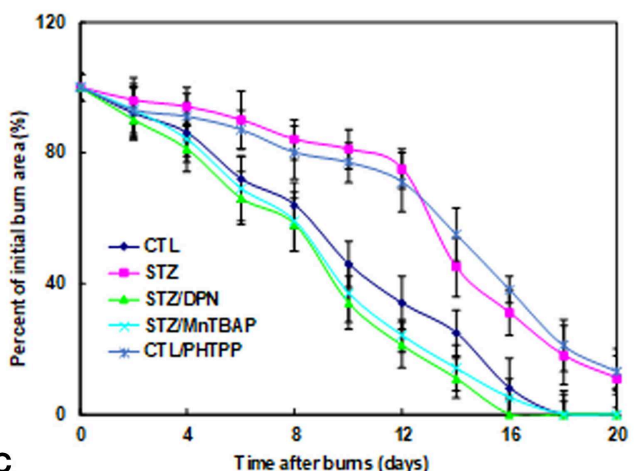

D

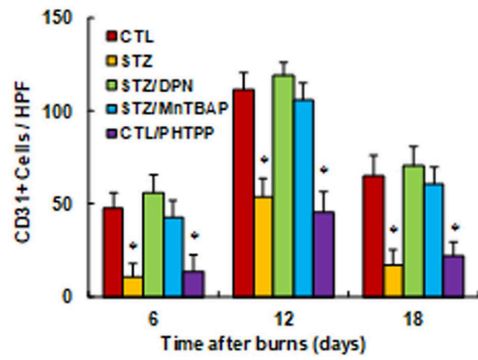

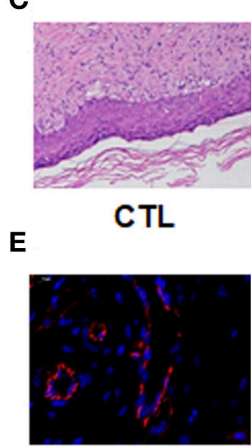

CTL

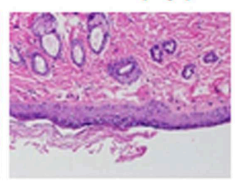

STZ

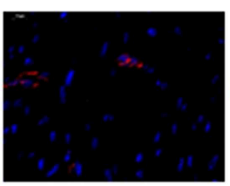

STZ

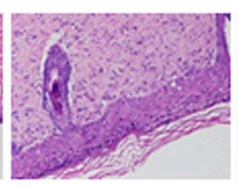

STZIDPN

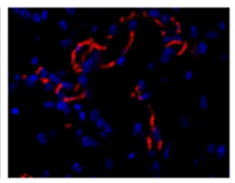

STZIDPN

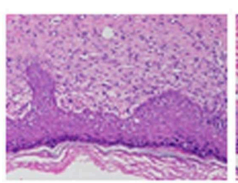

STZ/MnTBAP

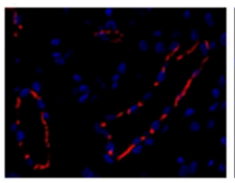

STZ/MnTBAP

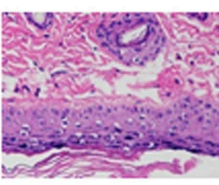

CTL/PHTPP

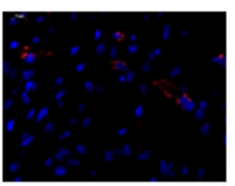

CTL/PHTPP

FIGURE 5 | ER $\beta$ activation accelerates wound healing in diabetic rats. The experimental rats were divided into 5 groups: control (CTL), diabetic (STZ) treatment, STZ rats treated by $450 \mu \mathrm{g} / \mathrm{kg} /$ day of DPN (STZ/DPN), STZ rats treated by $10 \mathrm{mg} / \mathrm{kg} /$ day of MnTBAP (STZ/MnTBAP), or CTL rats treated by $450 \mu \mathrm{g} / \mathrm{kg} / \mathrm{day}$ of PHTPP (CTL/PHTPP), for further analysis. (A) Photographs of representative wounds on day 12 after burns. (B) Graphical depiction of wound area in different days after burns. (C) H\&E stains of wound tissue on day 12 after burns with occurrence of granulation tissue in the wounds. (D) Numbers of CD31 positive vessels per HPF area on day 12 after burns. (E) The representative pictures for the evaluation of vascularity (assessed by CD31 immunohistochemistry) on day 12 after burns. $N=7$. ${ }^{*} P<0.05$, vs. CTL group. Data are expressed as mean \pm SEM.

mechanism for hyperglycemia-induced ER $\beta$ suppression is still unclear. We have previously showed that SIRT1 regulates ER $\beta$ suppression in endothelium and contributes to vascular aging (15). Hyperglycemia-induced decreased SIRT1 activity may potentially suppress ER $\beta$ expression $(31,32)$.

\section{Potential Effect of ER $\beta$ Expression in Diabetic Wound Healing}

In this study, we reported that $\operatorname{ER} \beta$ suppression contributes to hyperglycemia-induced persistent oxidative stress, and subsequently results in delayed diabetic wound healing. Activation of ER $\beta$ may break this ROS generation loop and accelerate wound healing. On the other hand, it has also been reported that $\operatorname{ER} \beta$ regulates the basal expression of $\operatorname{ERR} \alpha$ (22), and subsequently regulates nitric oxide (NO) generation via modulation of eNOS (33), together with lipid metabolism and mitochondrial function $(14,15,34,35)$. In this case, the potential effect of $\operatorname{ER} \beta$ activation on the treatment of diabetic wound healing should be a complicated process. Development of a more specific drug for delayed diabetic wound healing based on ER $\beta$ activation with fewer side effects should be in our considerations.

\section{CONCLUSIONS}

Taken altogether, our results show that hyperglycemia-induced ROS generation suppresses ER $\beta$ expression and subsequently results in SOD2 suppression with further elevated ROS generation. This forms a positive feed-forward loop for ROS generation and delays diabetic wound healing. ER $\beta$ agonist DPN, or SOD mimetic MnTBAP, breaks this ROS generation loop, ameliorates the oxidative stress-mediated pathological responses, and subsequently accelerates diabetic wound healing. This provides a potential targeting strategy for diabetic wound healing based on $\operatorname{ER} \beta$ activation. 


\section{ETHICS STATEMENT}

The animal protocol conformed to US NIH guidelines (Guide for the Care and Use of Laboratory Animals, No. 85-23, revised 1996), and was reviewed and approved by the Institutional Animal Care and Use Committee from Wuhan University.

\section{AUTHOR CONTRIBUTIONS}

PY wrote the paper. PY, LL, and WX designed, analyzed the data and interpreted the experiments. $\mathrm{LZ}$ and $\mathrm{HZ}$ performed vector constructions and gene expression analysis. QR, ZC, ZY, and XH performed statistical analysis and part of the rats experiments. $\mathrm{XZ}, \mathrm{ML}$, and MX performed the remaining experiments. All authors read and approved the final manuscript.

\section{REFERENCES}

1. Dhall S, Do DC, Garcia M, Kim J, Mirebrahim SH, Lyubovitsky J, et al. Generating and reversing chronic wounds in diabetic mice by manipulating wound redox parameters. J Diabetes Res. (2014) 2014:562625. doi: $10.1155 / 2014 / 562625$

2. Yuniarti AR, Setianto F, Marcellinus A, Hwang HJ, Choi SW, Trayanova N, et al. Effect of KCNQ1 G229D mutation on cardiac pumping efficacy and reentrant dynamics in ventricles: Computational study. Int J Numer Method Biomed Eng. (2018) 34:e2970. doi: 10.1002/cnm.2970

3. Kasbekar PU, Goel P, Jadhav SP. A decision tree analysis of diabetic foot amputation risk in Indian patients. Front Endocrinol. (2017) 8:25. doi: $10.3389 /$ fendo.2017.00025

4. Falanga V. Wound healing and its impairment in the diabetic foot. Lancet. (2005) 366:1736-43. doi: 10.1016/S0140-6736(05)67700-8

5. Long M, Rojo de la Vega M, Wen Q, Bharara M, Jiang T, Zhang R, et al. An essential role of NRF2 in diabetic wound healing. Diabetes. (2016) 65:78093. doi: 10.2337/db15-0564

6. Singh N, Armstrong DG, and Lipsky BA. Preventing foot ulcers in patients with diabetes. I Am Med Assoc. (2005) 293:21728. doi: 10.1001/jama.293.2.217

7. Zheng Z, Chen H, Li J, Li T, Zheng B, Zheng Y, et al. Sirtuin 1-mediated cellular metabolic memory of high glucose via the LKB1/AMPK/ROS pathway and therapeutic effects of metformin. Diabetes. (2012) 61:21728. doi: $10.2337 / \mathrm{db} 11-0416$

8. Liao Y, Gou L, Chen L, Zhong X, Zhang D, Zhu H, et al. NADPH oxidase 4 and endothelial nitric oxide synthase contribute to endothelial dysfunction mediated by histone methylations in metabolic memory. Free Radic Biol Med. (2018) 115:383-94. doi: 10.1016/j.freeradbiomed.2017.12.017

9. Patel H, Chen J, Das KC, and Kavdia M. Hyperglycemia induces differential change in oxidative stress at gene expression and functional levels in HUVEC and HMVEC. Cardiovasc Diabetol. (2013) 12:142. doi: 10.1186/1475-2840-12-142

10. Ohashi M, Runge MS, Faraci FM, Heistad DD. MnSOD deficiency increases endothelial dysfunction in ApoE-deficient mice. Arterioscler Thromb Vasc Biol. (2006) 26:2331-6. doi: 10.1161/01.ATV.0000238347.77590.c9

11. El Assar M, Angulo J, and Rodriguez-Manas L. Oxidative stress and vascular inflammation in aging. Free Radic Biol Med. 65:380-401. doi: 10.1016/j.freeradbiomed.2013.07.003

12. Liu Z, Gou Y, Zhang H, Zuo H, Zhang H, Liu Z, et al. Estradiol improves cardiovascular function through up-regulation of SOD2 on vascular wall. Redox Biol. (2014) 3:88-99. doi: 10.1016/j.redox.2014.11.001

13. Infante $\mathrm{T}$, Forte $\mathrm{E}$, Aiello $\mathrm{M}$, Salvatore $\mathrm{M}$, Cavaliere C. In vivo and in vitro analysis in coronary artery disease related to type 2 diabetes. Front Endocrinol. (2017) 8:209. doi: 10.3389/fendo.2017.00209

14. Zhan Y, Liu Z, Li M, Ding T, Zhang L, Lu Q, et al. ERbeta expression in the endothelium ameliorates ischemia/reperfusion-mediated

\section{FUNDING}

This study was financially supported by The National Natural Science Foundation of China, Project \#: 81772097 \& 81501667; Hubei Science \& Technology Development Project \#: 2016CFB473 \& 2016CFB589; Clinical Research Project from Health and Family Planning Commission of Wuhan Municipality: \# WX17A03; Bureau of Public Health of Hainan Province Key Project \#: 14A110065.

\section{SUPPLEMENTARY MATERIAL}

The Supplementary Material for this article can be found online at: https://www.frontiersin.org/articles/10.3389/fendo. 2019.00499/full\#supplementary-material

oxidative burst and vascular injury. Free Radic Biol Med. (2016) 96:22333. doi: 10.1016/j.freeradbiomed.2016.04.029

15. Kong D, Zhan Y, Liu Z, Ding T, Li M, Yu H, et al. SIRT1-mediated ERbeta suppression in the endothelium contributes to vascular aging. Aging Cell. (2016) 15:1092-102 doi: 10.1111/acel.12515

16. Zou Y, Lu Q, Zheng D, Chu Z, Liu Z, Chen H, et al. Prenatal levonorgestrel exposure induces autism-like behavior in offspring through ERbeta suppression in the amygdala. Mol Autism. (2017) 8:46. doi: 10.1186/s13229-017-0159-3

17. Campbell L, Emmerson E, Davies F, Gilliver SC, Krust A, Chambon P, et al. Estrogen promotes cutaneous wound healing via estrogen receptor beta independent of its antiinflammatory activities. J Exp Med. (2010) 207:182533. doi: $10.1084 /$ jem. 20100500

18. Ronconi KS, Stefanon I, Ribeiro Junior RF. Tributyltin and vascular dysfunction: the role of oxidative stress. Front Endocrinol. (2018) 9:354. doi: 10.3389/fendo.2018.00354

19. Muscoli C, Cuzzocrea S, Ndengele MM, Mollace V, Porreca F, Fabrizi F, et al. Therapeutic manipulation of peroxynitrite attenuates the development of opiate-induced antinociceptive tolerance in mice. J Clin Invest. (2007) 117:3530-9. doi: 10.1172/JCI32420

20. Batinic-Haberle I, Cuzzocrea S, Reboucas JS, Ferrer-Sueta G, Mazzon E, Di Paola R, et al. Pure MnTBAP selectively scavenges peroxynitrite over superoxide: comparison of pure and commercial MnTBAP samples to MnTE2-PyP in two models of oxidative stress injury, an SOD-specific Escherichia coli model and carrageenan-induced pleurisy. Free Radic Biol Med. (2009) 46:192-201. doi: 10.1016/j.freeradbiomed.2008.09.042

21. Bodnar AG, Ouellette M, Frolkis M, Holt SE, Chiu CP, Morin GB, et al. Extension of life-span by introduction of telomerase into normal human cells. Science. (1998) 279:349-52. doi: 10.1126/science.279.5349.349

22. Li H, Liu Z, Gou Y, Yu H, Siminelakis S, Wang S, et al. Estradiol mediates vasculoprotection via ERRalpha-dependent regulation of lipid and ROS metabolism in the endothelium. J Mol Cell Cardiol. (2015) 87:92101. doi: 10.1016/j.yjmcc.2015.08.008

23. Zhang H, Li L, Li M, Huang X, Xie W, Xiang W, et al. Combination of betulinic acid and chidamide inhibits acute myeloid leukemia by suppression of the HIF1alpha pathway and generation of reactive oxygen species. Oncotarget. (2017) 8:94743-58. doi: 10.18632/oncotarget.21889

24. Yao D, Shi W, Gou Y, Zhou X, Yee Aw T, Zhou Y, et al. Fatty acid-mediated intracellular iron translocation: a synergistic mechanism of oxidative injury. Free Radic Biol Med. (2005) 39:1385-98. doi: 10.1016/j.freeradbiomed.2005.07.015

25. Yao D, Vlessidis AG, Gou Y, Zhou X, Zhou Y, and Evmiridis NP. Chemiluminescence detection of superoxide anion release and superoxide dismutase activity: modulation effect of Pulsatilla chinensis. Anal Bioanal Chem. (2004) 379:171-7. doi: 10.1007/s00216-004-2527-z

26. Neal-Perry G, Yao D, Shu J, Sun Y, Etgen AM. Insulin-like growth factorI regulates LH release by modulation of kisspeptin and NMDA-mediated 
neurotransmission in young and middle-aged female rats. Endocrinology. (2014) 155:1827-37. doi: 10.1210/en.2013-1682

27. Thangarajah H, Yao D, Chang EI, Shi Y, Jazayeri L, Vial IN, et al. The molecular basis for impaired hypoxia-induced VEGF expression in diabetic tissues. Proc Natl Acad Sci U S A. (2009) 106:13505-10. doi: 10.1073/pnas.0906670106

28. Savoia P, Raina G, Camillo L, Farruggio S, Mary D, Veronese F, et al. Anti-oxidative effects of 17 beta-estradiol and genistein in human skin fibroblasts and keratinocytes. J Dermatol Sci. (2018) 92:62-77. doi: 10.1016/j.jdermsci.2018.07.007

29. Chatzikyrkou C, Bahlmann FH, Sushakova N, Scurt FG, Menne J, Nawroth $\mathrm{P}$, et al. Low-dose erythropoietin promotes wound-healing of ulcers in diabetics: Evidence from a phase-IIa clinical study. Diab Metab. (2016) 42:466-70. doi: 10.1016/j.diabet.2016.05.010

30. Vouillarmet J, Bourron O, Gaudric J, Lermusiaux P, Millon A, Hartemann A. Lower-extremity arterial revascularization: Is there any evidence for diabetic foot ulcer-healing? Diab Metab. (2016) 42:4-15. doi: 10.1016/j.diabet.2015.05.004

31. Zhang Y, Thai K, Jin T, Woo M, Gilbert RE. SIRT1 activation attenuates alpha cell hyperplasia, hyperglucagonaemia and hyperglycaemia in STZ-diabetic mice. Sci Rep. (2018) 8:13972. doi: 10.1038/s41598-018-32351-z

32. Shi X, Pi L, Zhou S, Li X, Min F, Wang S, et al. Activation of sirtuin 1 attenuates high glucose-induced neuronal apoptosis by deacetylating p53. Front Endocrinol. (2018) 9:274. doi: 10.3389/fendo.2018.00274
33. Sumi D, and Ignarro LJ. Estrogen-related receptor alpha 1 up-regulates endothelial nitric oxide synthase expression. Proc Natl Acad Sci USA. (2003) 100:14451-6. doi: 10.1073/pnas.2235590100

34. Kelly DP, and Scarpulla RC. Transcriptional regulatory circuits controlling mitochondrial biogenesis and function. Genes Dev. (2004) 18:357-68. doi: 10.1101/gad.1177604

35. Schreiber SN, Emter R, Hock MB, Knutti D, Cardenas J, Podvinec $M$, et al. The estrogen-related receptor alpha (ERRalpha) functions in PPARgamma coactivator 1alpha (PGC-1alpha)-induced mitochondrial biogenesis. Proc Natl Acad Sci USA. (2004) 101:6472-7. doi: 10.1073/pnas. 0308686101

Conflict of Interest Statement: The authors declare that the research was conducted in the absence of any commercial or financial relationships that could be construed as a potential conflict of interest.

Copyright (c) 2019 Zhou, Li, Xiao, Ruan, Chu, Ye, Zhong, Zhang, Huang, Xie, Li and Yao. This is an open-access article distributed under the terms of the Creative Commons Attribution License (CC BY). The use, distribution or reproduction in other forums is permitted, provided the original author(s) and the copyright owner(s) are credited and that the original publication in this journal is cited, in accordance with accepted academic practice. No use, distribution or reproduction is permitted which does not comply with these terms. 